\title{
Historia de la investigación marino-costera en Bahía Culebra, Pacífico Norte, Guanacaste, Costa Rica
}

\author{
Jorge Cortés 1,2 \\ 1. Centro de Investigación en Ciencias del Mar y Limnología (CIMAR), Ciudad de la Investigación, Universidad de \\ Costa Rica, San Pedro, 11501-2060 San José, Costa Rica; jorge.cortes@ucr.ac.cr \\ 2. Escuela de Biología, Universidad de Costa Rica, San Pedro, 11501-2060 San José, Costa Rica
}

Recibido 07-III-2011. Corregido 16-VI-2011. Aceptado 15-II-2012.

\begin{abstract}
History of coastal-marine research in Bahía Culebra, North Pacific, Guanacaste, Costa Rica. Bahía Culebra (Culebra Bay) is located on the north Pacific coast of Costa Rica in a seasonal upwelling area. In this paper I present the history of marine research at Bahía Culebra, to summarize what is known and to point out research that should be carried out. Marine organisms of the Bay have been studied since the 1920's and very intensely during the 1930's, with the Alan Hancock Pacific Expeditions and the New York Society Zoological Expeditions. Most marine research has been done since 1980 by the Marine Science and Limnology Reseach Center (Centro de Investigación en Ciencias del Mar y Limnología, CIMAR) at the University of Costa Rica. The best studied systems are the coral communities and reefs, followed by zooplankton research. In a compilation of published records of marine organisms 577 are reported and in this Special Issue 20 more are added, for a total of 597 marine species. Even so, more research is needed in several ecosystems and groups. It is necessary and important to develop managment plans to protect and conserve the marine ecosystems and biodiversity of Bahía Culebra. Rev. Biol. Trop. 60 (Suppl. 2): 19-37. Epub 2012 April 01.
\end{abstract}

Key words: Bahía Culebra, history of research, Costa Rica, marine environments, marine organisms.

Bahía Culebra está ubicada en la Provincia de Guanacaste en la costa Pacífico norte de Costa Rica $\left(10^{\circ} 36^{\prime} \mathrm{N}-85^{\circ} 39^{\prime} \mathrm{W}\right)$, y tiene una entrada de $2.1 \mathrm{~km}$ de ancho, $7 \mathrm{~km}$ de largo y la sección interna más ancha es de $5 \mathrm{~km}$. En la parte interna hay profundidades de hasta $30 \mathrm{~m}$, por lo que, junto con su condición de poco oleaje y relativa protección por las colinas circundantes, facilita el anclaje de barcos de gran calado (Fradin 1892 en Meléndez 1974, Beebe 1942a). Bahía Culebra ha sido una región muy visitada por expediciones y científicos desde el siglo XVII (Savage 2002). A partir de inicios del Siglo XX y hasta la presente se desarrollan investigaciones marinas en ella. Bahía Culebra también es uno de las regiones de mayor desarrollo turístico en Costa Rica.

En este trabajo hago una recapitulación de las investigaciones marinas desarrolladas en
Bahía Culebra, analizo los temas investigados y apunto a vacíos del conocimiento, algunos muy importantes para el manejo adecuado de los ambientes marinos de la Bahía.

\section{CRONOLOGÍA DE LAS INVESTIGACIONES MARINAS}

El primer organismos marino que se menciona de Bahía Culebra, es la culebra de mar, Pelamis platurus, que le da el nombre a la bahía, Bahía de las Culebras, y fue por Gonzalo Fernández de Oviedo (1535, en Taylor 1953). La primera publicación científica encontrada sobre organismos o ambientes marinos de Bahía Culebra es por Hermann Augener, quien vivió de 1872 a 1938 y fue curador de anélidos del Museo de Hamburgo en 
Alemania (Obituary: Nature 141: 863, 14 May 1938). Augener (1922) informa de la especies del anélido poliqueto Chloeia pseudeuglochis recolectada en la Bahía (Cuadro 1). Harlan K. Dean (com. pers. 2010) me ha indicado que el espécimen descrito por Augener estaba depositado en el Museo de Copenhague y podría haber sido recolectado (Augener no lo indica) por el botánico danés Anders Sandoe Oersted durante sus extensos viajes entre 1845 y 1848 por Centro América, en especial Costa Rica y Nicaragua. Pese a que Augener no ilustró el espécimen, por la descripción que brinda hace pensar a Dean (2004) que probablemente se trata de la especie Chloeia pinnata.

Las siguientes muestras de Bahía Culebra fueron recolectadas en 1933 con el barco U.S.S. Hannibal y depositadas en la colección del Instituto Oceanográfico Scripps (La Jolla, California, EUA). Este era un barco de la marina de los Estados Unidos de América que visitó Costa Rica en repetidas ocasiones entre 1933 y 1937. Recolectaron cinco núcleos de sedimentos, uno frente a Playa Hermosa en Bahía Culebra, dos por Quepos, uno mar afuera frente a Quepos y otro mar afuera al sur de la Isla del Caño (Bandy \& Arnal 1957). Diez y siete especies son reportadas para Bahía Culebra por Bandy y Arnal (1957) en su trabajo sobre foraminíferos recientes de la costa oeste de América Central (Cuadro 1).

La expedición a Bahía Culebra de la Fundación Allan Hancock a bordo del Velero III en 1934, es la que ha producido el mayor número de publicaciones sobre la mayor cantidad de grupos de organismos marinos (Cuadro 1). Fraser (1943a) proporciona una introducción a las expediciones de la Fundación Allan Hancock al Pacífico Oriental realizadas entre 1931 y 1941. Fraser (1943b) presenta el detalle de los sitios que fueron muestreados durante las expediciones y contiene una descripción de Bahía Culebra visitada brevemente del 12 al 13 de marzo de 1933 y del 24 al 25 de febrero de 1934. Incluye además dos fotografías, una de la Bahía desde un rodal de cactus y otra que parece ser la boca del estero Iguanita en la parte interna de la Bahía. Finalmente, Fraser (1943c) incluye los mapas con los sitios de muestreo: tres estaciones en 1933 (una en Playas del Coco y dos en Playa Panamá) y seis en 1934 (dos frente a la entrada de la Bahía y el resto en la boca y adentro).

La primera publicación de las expediciones de la Fundación Allan Hancock es por Drouet (1936) quien describe dos especies de cianobacterias recolectadas por William R. Taylor durante la expedición de 1934. Posteriormente, Taylor (1945) describe dos especies más de cianobacterias y 15 especies de algas entre verdes, rojas y pardas. Los siguientes trabajos publicados fueron sobre parásitos de peces, Wilson (1937) describe dos copépodos parásitos y Meserve (1938) un trematodo, a continuación, Manter (1940) describe cuatro especies más de tremátodos y finalmente Van Cleave (1940) describe dos acantocéfalos. Fraser (1938) describe 11 especies de hidroides bentónicos, Cushman y McCulloch (1939, 1940, 1942, 1948) 10 de foraminíferos, Lalicker y McCulloch (1940) tres más de foraminíferos, Hartmann (1940, 1944a, b) tres de poliquetos, Ziesenhenne (1940) una especie de ofiúrido, Osburn $(1950,1952)$ y Osburn y Soule (1953) nueve de briozoarios, Rost (1955) dos bivalvos, Soot-Ryen (1955) siete bivalvos más y Deichmann (1958) cuatro especies de holotúridos. La mayor cantidad de publicaciones son sobre crustáceos; Schmitt (1940) incluye el informe nuevo de una especie de estomatópodo, Garth (1940, 1958) describe 25 de cangrejos, Barnard (1954) y Barnard y Barnard (1982) dos de anfípodos, Haig (1960) 11 cangrejos más, Türkay (1970) otro cangrejo, Childs (1979) un picnogónido, Fritzsche (1980) un pez, McLaughlin (1981) un cangrejo ermitaño, Wicksten (1983) un camarón y Castro (1996) dos de cangrejos simbiontes de corales.

Otra expedición importante que visitó Bahía Culebra fue la Número 26 de la Sociedad Zoológica de Nueva York, a bordo del Yate Zaca del 24 al 31 de enero de 1938 (Beebe 1938). La expedición fue dirigida por William Beebe, uno de los naturalistas más sobresalientes del Siglo XX (Gould 2004). Posteriormente Beebe (1942a) publica un libro sobre esa expedición, 
CUADRO 1

Expediciones, instituciones o individuos que publicaron sobre especies marinas recolectadas en Bahía Culebra, Costa Rica. Se indica la referencia, el grupo taxonómico y la cantidad de especies de cada grupo

TABLE 1

Expeditions, institutions and individuals that published on marine species collected at Bahía Culebra, Costa Rica. The reference, taxonomic group and number os species of each group are indicated

\begin{tabular}{|c|c|c|c|}
\hline Expedición/Institución & Referencia & Grupo taxonómico & \# spp. \\
\hline Colección de museo & Augener 1922 & Poliqueto & 1 \\
\hline AHPE & Drouet 1936 & Cianobacterias & 2 \\
\hline AHPE & Rathbun 1937 & Crustáceos & 10 \\
\hline AHPE & Wilson 1937 & $\begin{array}{l}\text { Copépodo parásito } \\
\text { Pez }\end{array}$ & $\begin{array}{l}2 \\
1\end{array}$ \\
\hline AHPE & Fraser 1938 & Hidroides & 11 \\
\hline AHPE & Meserve 1938 & Tremátodo & 1 \\
\hline AHPE & Cushman \& McCulloch 1939 & Foraminíferos & 2 \\
\hline NYZS & Clark 1940 & Erizo de mar & 1 \\
\hline AHPE & Cushman \& McCulloch 1940 & Foraminífero & 1 \\
\hline AHPE & Garth 1940 & Cangrejo & 1 \\
\hline AHPE & Hartman 1940 & Poliqueto & 1 \\
\hline AHPE & Lalicker \& McCulloch 1940 & Foraminíferos & 3 \\
\hline AHPE & Manter 1940 & $\begin{array}{l}\text { Tremátodos parásitos } \\
\text { Peces }\end{array}$ & $\begin{array}{l}4 \\
2\end{array}$ \\
\hline AHPE & Schmitt 1940 & Estomatópodo & 1 \\
\hline AHPE & Van Cleave 1940 & Acantocéfalos & 2 \\
\hline AHPE & Ziesenhenne 1940 & Ofiúrido & 1 \\
\hline NYZS & Crane 1941a & Cangrejos & 1 \\
\hline NYZS & Crane 1941b & Cangrejos & 2 \\
\hline NYZS & Beebe $1942 b$ & Pez & 1 \\
\hline AHPE & Cushman \& McCulloch 1942 & Foraminíferos & 2 \\
\hline NYZS & Hertlein \& Strong 1943 & Bivalvos & 9 \\
\hline AHPE & Hartman 1944a & Poliqueto & 1 \\
\hline AHPE & Hartman 1944b & Poliqueto & 1 \\
\hline AHPE & Taylor 1945 & $\begin{array}{l}\text { Cianobacterias } \\
\text { Algas }\end{array}$ & $\begin{array}{c}2 \\
15\end{array}$ \\
\hline NYZS & Hertlein \& Strong 1946a & Bivalvos & 4 \\
\hline NYZS & Hertlein \& Strong 1946b & Bivalvo & 1 \\
\hline NYZS & Crane 1947 & Cangrejos & 17 \\
\hline NYZS & Hertlein \& Strong 1947 & Bivalvos & 4 \\
\hline AHPE & Clark 1948 & Erizos de mar & 2 \\
\hline AHPE & Fraser 1948 & Hidroide & 1 \\
\hline NYZS & Hertlein \& Strong 1948 & Bivalvos & 11 \\
\hline AHPE & Cushman \& McCulloch 1948 & Foraminíferos & 3 \\
\hline CAS & Hanna \& Strong 1949 & Gasterópodos & 10 \\
\hline NYZS & Hertlein \& Strong 1949a & Bivalvos & 3 \\
\hline NYZS & Hertlein \& Strong 1949b & Bivalvos & 3 \\
\hline NYZS & Hertlein \& Strong 1950 & Bivalvos & 6 \\
\hline
\end{tabular}


CUADRO 1 (Continuación)

Expediciones, instituciones o individuos que publicaron sobre especies marinas recolectadas en Bahía Culebra,

Costa Rica. Se indica la referencia, el grupo taxonómico y la cantidad de especies de cada grupo

TABLE 1 (Continued)

Expeditions, institutions and individuals that published on marine species collected at Bahía Culebra, Costa Rica. The reference, taxonomic group and number os species of each group are indicated

\begin{tabular}{|c|c|c|c|}
\hline Expedición/Institución & Referencia & Grupo taxonómico & \# spp. \\
\hline AHPE & Osburn 1950 & Briozoarios & 2 \\
\hline NYZS & Hertlein \& Strong 1951 & Gasterópodos & 3 \\
\hline AHPE & Osburn 1952 & Briozoarios & 6 \\
\hline AHPE & Osburn \& Soule 1953 & Briozoario & 1 \\
\hline Independiente & Taylor 1953 & Reptil & 1 \\
\hline AHPE & Barnard 1954 & Anfípodo & 1 \\
\hline AHPE & Rost 1955 & Bivalvos & 2 \\
\hline AHPE & Soot-Ryen 1955 & Bivalvos & 7 \\
\hline $\mathrm{SIO}$ & Bandy \& Arnal 1957 & Foraminíferos & 17 \\
\hline Independiente & Dawson 1957 & Algas & 3 \\
\hline CAS & Berry 1958 & Gasterópodo & 1 \\
\hline AHPE & Deichmann 1958 & Pepinos de mar & 4 \\
\hline AHPE & Garth 1958 & Cangrejos & 24 \\
\hline $\mathrm{BFE}$ & Dawson \& Beaudette 1959 & $\begin{array}{l}\text { Algas } \\
\text { Bivalvo } \\
\text { Gasterópodo }\end{array}$ & $\begin{array}{l}4 \\
1 \\
1\end{array}$ \\
\hline NYZS & Garth 1959 & Cangrejo & 1 \\
\hline AHPE & Haig 1960 & Cangrejos & 11 \\
\hline Independiente & Dawson 1960 & Algas & 9 \\
\hline Independiente & Dawson 1961 & Algas & 3 \\
\hline NYZS & Garth 1961 & Cangrejos & 6 \\
\hline NYZS & Garth 1966 & Cangrejos & 3 \\
\hline Colección de museo & Henry \& McLaughlin 1967 & Cirripedio & 1 \\
\hline NYZS & Haig 1968 & Cangrejos & 3 \\
\hline Independiente & Tsuda 1968 & Algas & 5 \\
\hline AHPE & Türkay 1970 & Cangrejo & 1 \\
\hline NYZS & Manning 1971 & Estomatópodos & 3 \\
\hline Colección de museo & McLean \& Poorman 1971 & Gasterópodo & 1 \\
\hline Colección de museo & Crane 1975 & Cangrejo & 1 \\
\hline Colección de museo & Henry \& McLaughlin 1975 & Cirripedios & 2 \\
\hline Colección de museo & Cernohorsky 1976 & Gasterópodo & 1 \\
\hline Colección de museo & Childs 1979 & Picnogónido & 1 \\
\hline Colección de museo & Fritzsche 1980 & Pez & 1 \\
\hline AHPE & McLaughlin 1981 & Cangrejo ermitaño & 1 \\
\hline AHPE & Barnard \& Barnard 1982 & Anfípodo & 1 \\
\hline Colección de museo & Gore 1982 & Cangrejos & 6 \\
\hline STRI & Glynn et al. 1983 & Corales & 2 \\
\hline AHPE & Wicksten 1983 & Camarón & 1 \\
\hline UNA & Madrigal-Castro et al. 1984 & Gasterópodo & 1 \\
\hline
\end{tabular}


CUADRO 1 (Continuación)

Expediciones, instituciones o individuos que publicaron sobre especies marinas recolectadas en Bahía Culebra,

Costa Rica. Se indica la referencia, el grupo taxonómico y la cantidad de especies de cada grupo

TABLE 1 (Continued)

Expeditions, institutions and individuals that published on marine species collected at Bahía Culebra, Costa Rica. The reference, taxonomic group and number os species of each group are indicated

\begin{tabular}{|c|c|c|c|}
\hline Expedición/Institución & Referencia & Grupo taxonómico & \# spp. \\
\hline CIMAR & Brusca \& Iverson 1985 & Isópodo & 1 \\
\hline CIMAR & Cortés \& Murillo 1985 & Corales & 7 \\
\hline Independiente & Crouch \& Poag 1987 & Foraminíferos & 5 \\
\hline Colección de museo & Kim \& Abele 1988 & Camarón pistola & 1 \\
\hline Literatura & Hendrickx \& Wicksten 1989 & Crustáceo & 1 \\
\hline Colección de museo & Jung 1989 & Gasterópodos & 6 \\
\hline Colección de museo & Hertz et al. 1992 & Gasterópodo & 1 \\
\hline Literatura & Wicksten \& Hendrickx 1992 & Crustáceos & 2 \\
\hline UNA & Cabrera-Peña et al. 1994 & $\begin{array}{l}\text { Plantas } \\
\text { Crustáceo }\end{array}$ & $\begin{array}{l}3 \\
1\end{array}$ \\
\hline UNA & Cabrera-Peña \& Solano-López 1996 & Crustáceo & 1 \\
\hline AHPE & Castro 1996 & Cangrejos & 2 \\
\hline CIMAR & Marques et al. 1996 & Platelmintos & 2 \\
\hline CIMAR & Jiménez 1997 & $\begin{array}{l}\text { Octocoral } \\
\text { Coral }\end{array}$ & $\begin{array}{l}1 \\
2\end{array}$ \\
\hline CIMAR, MZ-UCR & Cortés \& Guzmán 1998 & Corales & 6 \\
\hline CIMAR-PRB & Jiménez 1998 & Erizos de mar & 2 \\
\hline CIMAR & Hoberg et al. 1998 & Nemátodo & 1 \\
\hline FM, UCR & Pérez-Ponce de León et al. 1998 & $\begin{array}{l}\text { Platelmintos } \\
\text { Pez }\end{array}$ & $\begin{array}{l}2 \\
1\end{array}$ \\
\hline CIMAR-ISATEC & Alpermann 2001 & Pez & 1 \\
\hline CIMAR-ISATEC & Bednarski 2001 & $\begin{array}{l}\text { Ctenóforo } \\
\text { Copépodos }\end{array}$ & $\begin{array}{c}1 \\
39\end{array}$ \\
\hline CIMAR & Cortés 2001 & $\begin{array}{l}\text { Plantas } \\
\text { Alga } \\
\text { Gasterópodos } \\
\text { Bivalvo }\end{array}$ & $\begin{array}{l}2 \\
1 \\
2 \\
1\end{array}$ \\
\hline CIMAR & Morales-Ramírez et al. 2001 & Dinoflagelado & 1 \\
\hline Muchas fuentes & Skoglund 2001 & Bivalvos & 3 \\
\hline Colección de museo & Reid 2002 & Gasterópodo & 1 \\
\hline Muchas fuentes & Skoglund 2002 & Gasterópodos & 2 \\
\hline CIMAR & Ajtai et al. 2003 & Gasterópodo & 1 \\
\hline MZ-UCR, CIMAR & Breedy \& Guzman 2003 & Octocoral & 1 \\
\hline CIMAR & Suárez-Morales \& Morales-Ramírez 2003 & Copépodo & 1 \\
\hline MZ-UCR, CIMAR & Wehrtmann \& Vargas 2003 & Crustáceo & 1 \\
\hline CIMAR-ISATEC & Bednarski \& Morales-Ramírez 2004 & $\begin{array}{l}\text { Copépodos } \\
\text { Branquiópodo } \\
\text { Ostrácodo } \\
\text { Quetognato } \\
\text { Salpa }\end{array}$ & $\begin{array}{l}5 \\
1 \\
1 \\
1 \\
1\end{array}$ \\
\hline CIMAR & Rodríguez-Sáenz \& Rodríguez-Fonseca 2004 & Mamífero & 1 \\
\hline
\end{tabular}


CUADRO 1 (Continuación)

Expediciones, instituciones o individuos que publicaron sobre especies marinas recolectadas en Bahía Culebra, Costa Rica. Se indica la referencia, el grupo taxonómico y la cantidad de especies de cada grupo

TABLE 1 (Continued)

Expeditions, institutions and individuals that published on marine species collected at Bahía Culebra, Costa Rica. The reference, taxonomic group and number os species of each group are indicated

\begin{tabular}{|c|c|c|c|}
\hline Expedición/Institución & Referencia & Grupo taxonómico & \# spp. \\
\hline Colección de museo & Valdés \& Camacho-García 2004 & Gasterópodos & 7 \\
\hline GIACT-CIMAR & Vargas-Montero 2004 & $\begin{array}{l}\text { Cianobacteria } \\
\text { Diatomeas } \\
\text { Dinoflagelados }\end{array}$ & $\begin{array}{c}1 \\
3 \\
14\end{array}$ \\
\hline MZ-UCR, CIMAR & Breedy \& Guzmán 2005 & Octocorales & 1 \\
\hline CIMAR & Dominici-Arosemena et al. 2005 & Peces & 70 \\
\hline CIMAR & Fernández \& Cortés 2005 & Alga & 1 \\
\hline CIMAR & May-Collado \& Morales-Ramírez 2005 & Mamífero & 1 \\
\hline CIMAR & May-Collado et al. 2005 & Mamífero & 1 \\
\hline CIMAR & Spongberg 2006 & Sipuncúlidos & 2 \\
\hline MZ-UCR, CIMAR & Vargas \& Cortés 2006 & Cangrejos ermitaños & 2 \\
\hline MZ-UCR, CIMAR & Breedy \& Guzmán 2007 & Octocorales & 2 \\
\hline CIMAR & Alvarado 2008 & $\begin{array}{l}\text { Erizo de mar } \\
\text { Peces }\end{array}$ & $\begin{array}{l}1 \\
5\end{array}$ \\
\hline CIMAR & Fernández \& Alvarado 2008 & Algas & 3 \\
\hline MBCR, CIMAR & Bussing \& López 2009 & Pez & 1 \\
\hline MBCR, CIMAR & Castellanos et al. 2009 & Apendicularias & 4 \\
\hline MBCR, CIMAR & May-Collado 2009 & Mamífero & 1 \\
\hline MBCR, CIMAR & Rodríguez-Sáenz \& Gasca 2009 & Hidroides & 15 \\
\hline MBCR, CIMAR & Rodríguez-Sáenz \& Segura-Puertas 2009 & $\begin{array}{l}\text { Meduzoa: Hidrozoarios } \\
\text { Meduzoa: Medusas }\end{array}$ & $\begin{array}{c}48 \\
2\end{array}$ \\
\hline MZ-UCR, CIMAR & Breedy \& Guzman 2011 & Octocoral & 1 \\
\hline SBC, CIMAR & Samper-Villareal et al. 2012 & Mangles & 5 \\
\hline SBC, CIMAR & Vargas-Castillo 2012 & Crustáceos & 15 \\
\hline
\end{tabular}

Código:

$\mathrm{AHPE}=$ Allan Hancock Pacific Expedition.

$\mathrm{BFE}=$ Beaudette Foundation Expedition .

CAS = California Academy of Sciences, San Francisco, California, EUA.

CIMAR = Centro de Investigación en Ciencias del Mar y Limnología, Universidad de Costa Rica.

Colección de museo = Revisión de especimenes depositados en museos.

FM, UCR = Facultad de Microbiología, Universidad de Costa Rica.

GIACT = Maestría en Gestión Integrada de Áreas Costeras Tropicales, Universidad de Costa Rica.

Independiente $=$ Recolectó y trabajó las colecciones.

ISATEC = Internacional Studies in Aquatic Tropical Ecology, Universidad de Bremen, Bremen, Alemania.

Literatura $=$ Revisiones de registros publicados.

MBCR = Libro "Marine Biodiversity of Costa Rica, Central America" editado por I.S. Wehrtmann \& J. Cortés (2009).

Muchas fuentes = Literatura y colecciones de museos.

MZ-UCR = Museo de Zoología, Universidad de Costa Rica.

NYZS = New York Zoological Society, Bronx, Nueva York, EUA.

$\mathrm{PRB}=$ Posgrado Regional de Biología, Universidad de Costa Rica.

$\mathrm{SBC}=$ Suplemento Bahía Culebra, Revista de Biología Tropical, Volumen 60, Suplemento 2, 2012.

SIO = Scripps Institution of Oceanography, La Jolla, California, EUA.

STRI = Smithsonian Tropical Research Institute, Panamá.

UNA $=$ Universidad Nacional, Heredia, Costa Rica 
con un capítulo completo (Cap. XVII) dedicado a los días que permanecieron en la Bahía titulado "The Bay of Sea Snakes" (La bahía de las culebras de mar), el cual incluye una fotografía de una Pelamis platurus encallada en una playa. En la Bahía hicieron tres dragados sobre un fondo lodo-arenoso, cuatro colectas con luces (atrae organismos en las noches), además de recolecciones a mano en aguas someras y la zona de entremareas (Beebe 1938, 1942a). Algunas publicaciones importantes de esta expedición son las de Jocelyn Crane (1941a, b, 1947), William Beebe (1942b), John S. Garth $(1959,1961,1966)$ y Janet Haig (1968) sobre cangrejos, y la impresionante serie sobre bivalvos de Leo George Hertlein y Archibald McClure Strong (1943, 1946a, b, 1947, 1948, 1949a, b, 1950) (Cuadro 1).

Usando las colecciones de la Academia de Ciencias de California en San Francisco, California, G. Dallas Hanna \& Archibald McClure Strong (1949) informan de diez especies de gastrópodos del género Conus de Bahía Culebra. Posteriormente, S. Stillman Berry (1958) usando esas colecciones describe una nueva especie de caracol (Cuadro 1).

También hubo investigadores independientes que contribuyeron al conocimiento de la biodiversidad marina de Bahía Culebra (Cuadro1). Un ejemplo de estos es Elmer Y. Dawson, quien visitó Costa Rica en varias oportunidades: 1957, 1959 y 1962. En 1959 fue con la Expedición Beaudette al Pacífico, ocasión en recolectó en Bahía Culebra (Dawson 1957, 1960, 1961, 1962, Dawson \& Beaudette 1959). En enero de 1973, Anthony T. Tu recolecta varios miles de serpientes marinas, Pelamis platurus, para estudiar el patrón de coloración. La mayoría tenía un patrón tricolor, negro-amarillo-café, seguido por negro-amarillo y solamente 4 de las 3077 recolectadas eran totalmente amarillas. Además hizo extracciones del veneno (Tu 1976). También a inicios de 1973, Róger Bolaños y colaboradores recolectaron $P$. platurus, con un porcentaje alto de hembras grávidas, y definen más patrones de coloración, pero siempre las más abundantes son las que tiene tres o dos colores. Además hicieron extracciones de veneno y encontraron que tenía un efecto muy fuerte en ratones (Bolaños et al. 1974). Peter W. Glynn, en ese momento con el Smithsonian Tropical Research Institute (STRI) en Panamá, visita la región de Bahía Culebra en 1978 y publica el primer trabajo sobre arrecifes coralinos del Pacífico de Costa Rica, y el impacto que tuvo la Pequeña Edad de Hielo sobre esos arrecifes (Glynn et al. 1983).

En la década de 1980 científicos del Centro de Investigación en Ciencias del Mar y Limnología (CIMAR) de la Universidad de Costa Rica inicia sus investigaciones en Bahía Culebra, las que han continuado hasta el presente (Cuadro 1). Los primeros trabajos fueron sobre los arrecifes coralinos (Cortés \& Murillo 1985), otro en que se describen dos nuevas especies de parásitos céstodos de peces (Marques et al. 1996) y sobre el posible impacto de un derrame de petróleo sobre la zona costera (Acuña et al. 1996/1997). Los estudios sobre arrecifes y comunidades coralinas recibieron un fuerte impulso con las investigaciones de Carlos E. Jiménez Centeno a partir de finales de 1991 (Jiménez 1997, 1998, 2001a, b, Jiménez et al. 2001, 2010, Jiménez \& Cortés 2003). Sus trabajos sentaron la línea base de dichos ecosistemas, hecho que ha permitido evaluar el cambio experimentado producto del calentamiento de las aguas por El Niño (Jiménez et al. 2001). Se encontró que las tasas de crecimiento de la mayoría de las especies de corales en la Bahía son más altas que en otras partes del Pacífico Oriental, posiblemente porque estos corales aprovechan el alimento en suspensión durante el afloramiento y los productos de la fotosíntesis de las zooxantelas todo el año (Jiménez \& Cortés 2003). Estos son ecosistemas dinámicos que fueron impactados hace más de 300 años durante la Pequeña Edad de Hielo (Glynn et al. 1983) y que tienen una recuperación muy lenta, posiblemente por la reducida reproducción sexual de las especies de coral (Cortés \& Jiménez 2003, Bezy 2009). Actualmente están en preparación otros trabajos que evalúan impactos más recientes (C.E. Jiménez com. pers.).

A partir de 1997 se inicia un monitoreo mensual en Bahía Culebra, en el cual se miden 
parámetros físicos como la temperatura del agua y sedimentos en varios arrecifes y a diferentes profundidades, también se toman muestras de agua para la determinación de nutrimentos. Durante la década del 2000 se continua con el monitoreo y con las investigaciones de los corales y los sistemas coralinos de la Bahía (Cortés et al. 2010). Como parte del establecimiento de la línea base de parámetros ambientales, Gisèlle Muller-Parker y colaboradores tomaron datos sobre temperatura, salinidad, nutrimentos y atenuación de la luz a diferentes longitudes de onda en Bahía Culebra en enero 1997 (Muller-Parker \& Cortés 2001). Encontraron que la claridad del agua en Bahía Culebra es alta, al igual que las concentraciones de nutrimentos comparadas con las del Caribe, la otra región estudiada. En Bahía Culebra el fosfato es el nutrimento limitante.

Daniel Gateño y colaboradores publican la descripción de tumores en Pavona clavus; sin embargo, no se ha podido determinar el origen de esas deformaciones (Gateño et al. 2003). Ese mismo año, Cindy Fernández García comienza a estudiar la expansión del alga Caulerpa sertulariodes en Bahía Culebra y posteriormente publica un trabajo (Fernández \& Cortés 2005) y una tesis sobre el tema (Fernández-García 2007). Esta alga afecta las comunidades y arrecifes coralinos ya que reduce las tasas de crecimiento de los corales y en algunos casos llegan a matarlos; Bernadette $\mathrm{M}$. Bezy y colaboradores describe como la expansión de $C$. sertulariodes resultó en la muerte de una arrecife construido por corales del género Psammocora (Bezy et al. 2006).

Las densidades y concentraciones de pigmentos en las zooxantelas (dinoflagelados simbiontes de los corales constructores de arrecifes) del coral Pavona clavus fueron estudiadas entre el 2004 y el 2005, como parte de la Tesis de Maestría de Shinichi Sunagawa (2005, Sunagawa et al. 2008). Se encontró que la concentración de clorofila $a$ y $c$ disminuye al aumentar la intensidad lumínica y bajar las temperaturas, mientras que la densidad de zooxantelas se reduce al bajar las temperaturas, como sucede en la época de afloramiento estacional en Bahía Culebra. Se ha propuesto que los corales recurren a la alimentación heterotrófica durante el afloramiento (Sunagawa et al. 2008), lo cual podría explicar las tasas tan altas de crecimiento de corales en la Bahía (Jiménez \& Cortés 2003).

La reproducción sexual de corales del Pacífico Tropical Oriental ha sido estudiada por Peter W. Glynn y colaboradores (Glynn et al. 1991, 1994, 1996, 2000, 2008, 2011). Se ha encontrado que hay producción y liberación de gametos, sin embargo el reclutamiento es mínimo. La falta de reclutamiento se puede deber a que los gametos liberados son infértiles, que no hay fertilización, que no hay desarrollo del cigoto o embrión, que las larvas son depredadas, que no pueden asentarse por alguna razón o que los reclutas son depredados (Ritson-Williams et al. 2009). Bernadette M. Bezy estudió en detalle la reproducción sexual del coral Pavona clavus entre el 2004 y 2005, uno de los principales constructores de arrecifes coralinos en Bahía Culebra (Bezy 2009, Bezy et al. En prep.). Ella encontró que hay producción de gametos viables, fertilización y producción de larvas que se pueden asentar. El problema, y esto podría explicar el bajo nivel de reclutamiento sexual de los corales en el Pacífico Tropical Oriental, es que durante una parte del período reproductivo casi todas las colonias son hembras y después machos, con un período de traslape mínimo, reduciéndose significativamente las posibilidades de fertilización.

Ann M. Tarrant y colaboradores (Tarrant et al.2008) investigaron la presencia de receptores nucleares en el coral Pocillopora damicornis, recolectada en Bahía Culebra, tema del que se conoce muy poco en cnidarios. Encontraron tres receptores nucleares que podrían regular procesos fisiológicos: dos de ellos son similares a receptores de vertebrados importantes en la diferenciación neuronal, el tercero no se pudo relacionar a receptores nucleares conocidos, por lo que concluyen que son necesarios más estudios para esclarecer las funciones de esos receptores en el desarrollo y fisiología de corales. 
Jorge Cabrera-Peña y colaboradores de la Universidad Nacional, Heredia, Costa Rica, realizaron varias investigaciones sobre crustáceos en Bahía Culebra (Cuadro 1). MadrigalCastro et al. (1984) encontraron diferencias significativas en parámetros poblaciones al comparar dos poblaciones de Thais brevidentata, de dos zonas rocosas en Playa Panamá separadas poco más de un kilómetro. El cangrejo Ucides occidentalis en el manglar del estero de Playa Panamá, tiene un tamaño entre 36.7 y $84.5 \mathrm{~mm}$ y una proporción de sexos de casi dos machos por hembra (Cabrera-Peña et al. 1994). Cabrera-Peña \& Solano-López (1996) informan que el tamaño del cangrejo Pontonia margarita asociado al bivalvo de la zona rocosa al norte de Playa Panamá, Pinctada mazatlanica, fue de $6.39 \pm 2.02 \mathrm{~mm}$, la proporción de machos: hembras fue de 1:1.15 y en $93 \%$ de los bivalvos recolectados se hallaron parejas de cangrejos. Kelmo y Vargas (2002), del Museo de Zoología de la Universidad de Costa Rica incluyen una especie de hidroide de Bahía Culebra en su publicación sobre hidroides de Costa Rica.

Hoberg et al. (1998) describen la especie de nemátodo, Echinocephalus janzeni, parásito de la raya, Himantura pacifica, de Playa Panamá. Pérez-Ponce de León et al. (1998) informan de una larva de platihelmito tremátodo parásito de pez, Haemulon maculicauda, recolectados en Playa Hermosa. Indican otra especie recolectada en Playa Nacascolo, Bahía Prieta, Limón con las mismas coordenadas que Playa Hermosa, hay un error con esta ubicación. Finalmente, Rodríguez-Ortíz et al. (2004) presenta un listado de los helmintos parásitos de vertebrados en Costa Rica. En esta lista incluyen seis especies de digéneos (Plathyhelminthes), una de monogéo, dos de céstoda, dos de acantocéfala y una de nemátodo parásitos de peces en Bahía Culebra.

El erizo de mar Astropyga pulvinta, forma agregaciones de adultos en algunas regiones de Bahía Culebra durante el período de afloramiento estacional (diciembre a abril), aparentemente para explotar las algas que proliferan en esa época (Alvarado 2008). Juan José Alvarado encontró, además, que estas concentraciones de erizos sirven de refugio a muchas especies de peces que son extraídas para acuarios. Es importante proteger este erizo y monitorear sus poblaciones como también la de los peces asociados (Alvarado 2008).

Sobre peces se han realizado pocos trabajos. Las primeras menciones de especies de peces de Bahía Culebra son en trabajos sobre parásitos de peces (Wilson 1937, Manter 1940), posteriormente, William Beebe (1942) describe una especie cuyo nombre es modificado por Pfeiler (2008). Arturo Dominici-Arosemena (1999) para su tesis de Maestría en Biología de la Universidad de Costa Rica estudia la estructura poblacional de los peces de arrecifes de Bahía Culebra, con énfasis en las especies de mayor importancia comercial para la pesquería de peces ornamentales o de acuario. Informa de 75 especies en 28 familias de peces de arrecifes, siendo los planctívoros y comedores de invertebrados lo grupos más importantes (Dominici-Arosemena et al. 2005). Posteriormente, Tilman J. Alpermann (2001), del programa de cooperación del CIMAR con el Posgrado "Internacional Studies in Aquatic Tropical Ecology" (ISATEC, Universidad de Bremen, Alemania), estudia la pesquería de peces ornamentales, con énfasis en la dinámica poblacional del lábrido "Señorita arco iris", Thalassoma lucasanum. Encuentra que las poblaciones de peces ornamentales son bajas, indicando sobre explotación, además, las de $T$. lucasanum, muestran estructuras de población alteradas, por la extracción de los machos terminales. Sugiere que para conservar y en un futuro explotar sosteniblemente las poblaciones de peces ornamentales, se debe restringir más la pesca o empezar a hacer cierres parciales de esta pesquería.

Álvaro Morales Ramírez y colaboradores han informado de nuevas especies y de la presencia de especies tanto de zooplancton como de fitoplancton, previamente desconocidas en Bahía Culebra y en algunos casos de aguas costarricenses. Se describió una especie nueva de copépodo de la familia Monstrillidae, siendo este el primer informe para el Pacífico 
de Centroamérica de ese grupo de copépodos (Suárez-Morales \& Morales-Ramírez 2003). También hay registros nuevos como el dinoflagelado Lingulodinium polyedrum, el cual presentó una proliferación masiva en el 2000 en Bahía Culebra; especie asociada con la producción de toxinas paralizantes tales como saxitoxinas y yesotoxinas (Morales-Ramírez et al. 2001). Melinda K. Bednarski (2001, Bednarski \& Morales-Ramírez 2004), del Posgrado ISATEC, Universidad de Bremen, Alemania, estudió el zooplancton de Bahía Culebra, con énfasis en los copépodos en el año 2000. Ella informa de la presencia de al menos 39 especies de copépodos y de especies de ctenóforos, quetognatos, cladóceros y ostrácodos. Maribelle Vargas-Montero (2004) en su tesis sobre proliferaciones algales en Costa Rica, presenta una lista del fitoplancton que incluye una especie de cianobacteria, tres de diatomeas y 14 de dinoflagelados. Una de las contribuciones más significativas a la biodiversidad marina de Bahía Culebra es la tesis de Karina Rodríguez-Sáenz (2005) y sus publicaciones posteriores sobre zooplancton gelatinoso (Rodríguez-Sáenz \& Gasca 2009, Rodríguez-Sáenz \& Segura-Puertas 2009). Rodríguez-Sáenz y colaboradoras indican la presencia de 65 especies de medusas en Bahía Culebra (Cuadro 1).

Aunque Bahía Culebra ha sido estudiada desde hace muchas décadas y se ha informado en la literatura científica la presencia de 577 especies marinas (Cortés et al. 2012) la riqueza de especies debe ser mucho mayor.

En la síntesis sobre manglares del Pacífico Norte de Costa Rica, Priscilla Zamora-Trejos y Jorge Cortés (2009) comentan la necesidad de más investigaciones sobre este importante ecosistema. En Bahía Culebra solamente se han realizado estudios en el manglar de Playa Panamá, aunque hay manglares también en Iguanita. La extensión del manglar de Playa Panamá en 1998 era de 60 ha (Bravo \& Rivera 1998), sin embargo, no aparece delimitado en el mapa 1:50 000 del Instituto Geográfico Nacional de Costa Rica (Zamora-Trejos \& Cortés 2009).

Los estudios sobre contaminación marina son otra área de investigación importante en
Bahía Culebra. Dos proyectos de investigación coordinados por José A. Vargas, CIMAR, analizaron varias regiones del país, Moín en la costa Caribe, y en el Pacífico: Golfo de Nicoya, Golfo Dulce, Bahía Culebra y el Parque Nacional Isla del Coco. El primer proyecto, Contaminación Costera en Costa Rica (CoCosRi), desarrollado entre el 2000 y el 2003, con muestreos de campo entre el 2000 y el 2002. Dentro de este proyecto se encontró que los niveles de hidrocarburos de petróleo disueltos y dispersos en Bahía Culebra son tan bajos que no se detectaron con los métodos usados (Acuña-González et al. 2004). En el estudio de los metales traza, Bahía Culebra se encontraron los niveles promedio más bajos de Zinc y Plomo, y los más bajos absolutos de Cobre y Hierro (García-Céspedes et al. 2004). En cuanto a la contaminación fecal, los niveles más bajos de coliformes totales y coliformes fecales fueron en Bahía Culebra (García et al. 2006). Las concentraciones de bifenilos policlorados (BPCs) en sedimentos también fueron bajas en Bahía Culebra (Spongberg 2004).

El segundo proyecto sobre contaminación marina que incluyó Bahía Culebra coordinado por José A. Vargas, "Impacto de la contaminación en sistemas endocrinos de organismos marinos", se extiende del 2003 al 2006. Dentro de este proyecto Gravel et al. (2006) estudiaron el tema de imposexo (desarrollo de características sexuales masculinas en gasterópodos femeninos) con el caracol Thais brevidentata; Bahía Culebra fue usado como sitio de referencia por los niveles bajos de contaminación que presentaba (ver arriba). Spongberg (2006) analizó la presencia de BPCs en gusanos sipuncúlidos, encontrando las concentraciones más bajas en Bahía Culebra cuando se les compararon con las obtenidas en el Golfo de Nicoya y el Golfo Dulce.

Finalmente, Omar G. Lizano y colaboradores (Lizano et al. 2008) en su estudio sobre radionucleídos naturales y artificiales en sedimentos marinos de Bahía Culebra, realizado entre el 2001 y el 2005, encontraron que los isótopos $\mathrm{K}^{40}, \mathrm{Cs}^{137}, \mathrm{Bi}^{212}, \mathrm{~Pb}^{212}, \mathrm{Bi}^{214}$, $\mathrm{Pb}^{214}, \mathrm{Ra}^{226}, \mathrm{Ac}^{228}$ y $\mathrm{U}^{235}$ se encuentran en 
concentraciones más bajas que otras zonas del país. Las concentraciones determinadas son las esperadas de fuentes naturales de estos radionucleídos.

En 1995 Enrique Ibarra Gene defendió su tesis de Licenciatura en Economía (IbarraGene 1995) donde se analiza el valor de dos actividades antagónicas en la región (Jiménez 1997). Por un lado la extracción de peces ornamentales para acuarios y por otra la actividad de buceo recreativo. Ibarra-Gene (1995) encontró que había sobreexplotación de peces posiblemente por tres circunstancias: 1) controles inadecuados, 2) subarriendo ilegal de licencias, y 3 ) incentivos a la exportación que distorsiona el precio de mercado; problemas que todavía después de 15 años persisten.

\section{ESTADO DEL CONOCIMIENTO Y VACÍOS DE INVESTIGACIÓN}

Algunos ecosistemas de Bahía Culebra han sido bien estudiados, por ejemplo los arrecifes y comunidades coralinas (Jiménez 1997, 1998, 2001a, b, Jiménez et al. 2001, Jiménez \& Cortés 2003, Bezy et al. 2006) las cuales están siendo monitoreadas hasta la fecha. De los manglares hay un trabajo de hace muchos años sobre una especie de cangrejo (Cabrera-Peña et al. 1994) y en este suplemento se presenta una descripción detalladas de ellos (SamperVillareal et al. 2012). Los pastos marinos se deben re-evaluar para actualizar el trabajo de Cortés (2001). El plancton, especialmente el zooplancton ha sido bien estudiados por Karina Rodríguez-Sáenz. De la zona rocosa de entremareas hay dos publicaciones sobre un gasterópodo (Madrigal-Castro et al. 1984) y sobre la asociación de un crustáceo y un molusco (Cabrera-Peña \& Solano-López 1996), pero ninguna sobre la composición de las comunidades. No hay publicaciones sobre la fauna bentónica o sobre los organismos de las playas. Se ha evaluado en impacto del alga Caulerpa sertularioides (Fernández \& Cortés 2005, FernándezGarcía 2007) y se debe continuar su monitoreo a largo plazo. También se debe continuar con el monitoreo de contaminantes, especialmente por la alteración acelerada que se está dando en Bahía Culebra y su posible impacto en los organismos y ambientes marinos.

Sobre la biodiversidad, Cortés et al. (2012) informan de 577 especies basadas en registros publicados. A esto se le agregan cinco especies de mangle (Samper-Villareal et al. 2012) y 15 especies de crustáceos (Vargas-Castillo 2012) (Cuadro 1). Faltan trabajos sobre muchos otros grupos de animales, como son los poliquetos y se deben actualizar las listas de otros grupos.

Hacen falta más estudios sobre las diferentes pesquerías en Bahía Culebra y sus impactos, también el desarrollo de modelos tróficos, como el del Golfo Dulce (Wolff et al. 1996) y el Golfo de Nicoya (Wolff et al. 1998). Estos modelos son una herramienta importante para el manejo ecosistémico de las pesquerías. También faltan investigaciones detalladas sobre la circulación de las aguas en Bahía Culebra, como se ha hecho en el Golfo Dulce (Svendsen et al. 2006).

Bahía Culebra es una región de gran valor para Costa Rica, de gran interés histórico, arqueológico, económico, biológico y oceanográfico, por lo que se debe proteger y conservar. Es fundamental desarrollar una propuesta de ordenamiento terrestre y marino, y de protección y conservación de sus recursos naturales.

\section{AGRADECIMIENTOS}

Le agradezco profundamente a Kay Hale, ex-Directora de la biblioteca de RSMAS, Universidad de Miami, quien desde hace muchos años me consigue copias de trabajos antiguos y algunos muy difíciles de obtener. Al Smithsonian Tropical Research Institute en Panamá y al Museo Nacional de Historia Natural, Smithsonian Institution, Washington D.C. por el uso de sus excelentes bibliotecas. Este último lo pudo visitar gracias a una beca Fulbright Scholar. Harlan Dean ayudó con bibliografía, comentarios y datos, al igual que José A. Vargas y Rita Vargas. Le agradezco a Celeste Sánchez, José A. Vargas y Juan José Alvarado la revisión detallada del manuscrito. La Vicerrectoría de Investigación de la Universidad de Investigación ha 
financiado muchos de mis proyectos que me han puesto en contacto con la historia de la investigación marina en Costa Rica.

\section{RESUMEN}

Bahía Culebra está ubicada en la costa Pacífica norte de Costa Rica en una región de afloramiento estacional. En este trabajo presento la historia de la investigación marina en Bahía Culebra, para sintetizar lo que se ha hecho y resaltar lo que falta por investigar. Los organismos marinos de Bahía Culebra se empezaron a estudiar en la década de 1920 y muy intensamente en la década de 1930 con las expediciones de la Fundación Allan Hancock y de la Sociedad Zoológica de Nueva York. La mayor parte de la investigación marina se ha realizado desde la década de 1980 por investigadores y estudiantes del Centro de Investigación en Ciencias del Mar y Limnología (CIMAR) de la Universidad de Costa Rica. Los ecosistemas mejor estudiados son las comunidades y arrecifes coralinos, seguido por las investigaciones sobre zooplancton. Se han publicado registros de 577 especies marinas en Bahía Culebra y se le suman 20 especies más con las publicadas en este Suplemento. Aún así, todavía falta estudiar varios ecosistemas y grupos de organismos. Es imperativo realizar esfuerzo para proteger y conservar los ecosistemas y biodiversidad marina de Bahía Culebra.

Palabras clave: Bahía Culebra, historia de la investigación, Costa Rica, ambientes marinos, organismos marinos.

\section{REFERENCIAS}

Acuña, J., J. Cortés \& M. Murillo. 1996/1997. Mapa de sensibilidad ambiental para derrames de petróleo en las costas de Costa Rica. Rev. Biol. Trop. 44(3)/45(1): 463-470.

Acuña-González, J.A., J.A. Vargas-Zamora, E. GómezRamírez \& J. García-Céspedes. 2004. Hidrocarburos de petróleo, disueltos y dispersos, en cuatro ambientes costeros de Costa Rica. Rev. Biol. Trop. 52 (Supl. 2): $43-50$.

Ajtai, P., Y. Camacho-García \& I.S. Wehrtmann. 2003. Range extension of Crosslandia daedali (Nudibranchia: Scyllaeidae). Rev. Biol. Trop. 51: 274.

Alpermann, T.J. 2001. The fisheries of ornamental fishes in Guanacaste, Costa Rica with special emphasis on the population dynamics of the Cortez Rainbow wrasse, Thalassoma lucasanum (Gill 1863). Tesis de Maestría, Univ. Bremen. Bremen, Alemania. 84 p.

Alvarado. J.J. 2008. Seasonal occurrence and aggregation behavior of the sea urchin Astropyga pulvinata
(Echinodermata: Echinoidea) in Bahía Culebra, Costa Rica. Pac. Sci. 62: 579-592.

Augener, H. 1922. Ueber litorale Polychaeten von Westindien. Sitz. Ges. Naturf. Freunde Berlín 1922(3-5): 38-53.

Bandy, O.L. \& R.E. Arnal. 1957. Distribution of Recent Foraminifera off the west coast of Central America. Bull. Amer. Assoc. Petrol. Geol. 41: 2037-2053.

Barnard, J.L. 1954. Amphipoda of the family Ampelisceidae collected in the eastern Pacific by the Velero III and Velero IV. Allan Hancock Pac. Exped. 18: 1-137.

Barnard, J.L. \& C.M. Barnard. 1982. Revision of Foxiphalus and Eobrolgus (Crustacea: Amphipoda: Phoxocephalidae) from American Oceans. Smithson. Contr. Zool. 372: 1-35.

Bednarski, M. 2001. Macrozooplankton of Culebra Bay, Costa Rica, with an emphasis on copepods. Tesis de Maestría, Univ. Bremen. Bremen, Alemania. 155 p.

Bednarski, M. \& A. Morales-Ramírez. 2004. Composition, abundance and distribution of macrozooplankton in Culebra Bay, Gulf of Papagayo, Pacific coast of Costa Rica and its value as bioindicator of pollution. Rev. Biol. Trop. 52 (Suppl. 2): 105-118.

Beebe, W. 1938. Eastern Pacific expeditions of the New York Zoological Society, XIV. Introduction, itinerary, list of stations, nets and dredges of the eastern Pacific Zaca expedition, 1937-1938. Zoologica 23: 287-298.

Beebe, W. 1942a. Book of Bays. Barcourt, Brace and Company, New York. 302 p.

Beebe, W. 1942b. Eastern Pacific expeditions of the New York Zoological Society, XXX. Atlantic and Pacific fishes of the genus Dixonina. Zoologica 27: 43-48.

Berry, S.S. 1958. Notices of new eastern Pacific Mollusca - II. Leaflets Malacol. 1: 83-90.

Bezy, M.B. 2009. Reproducción sexual y reclutamiento del coral masivo, Pavona clavus, en Bahía Culebra, Golfo de Papagayo, Costa Rica. Tesis de Maestría en Biología, Universidad de Costa Rica. San Pedro, Costa Rica. 151 p.

Bezy, M.B., C. Jiménez, J. Cortés, A. Segura, A. León, J. J. Alvarado, C. Gillén \& E. Mejía. 2006. Contrasting Psammocora-dominated coral communities in Costa Rica, tropical eastern Pacific. Proc. 10th Int. Coral Reef Symp., Okinawa: 376-381. 
Bravo, J. \& L. Rivera. 1998. Mapas de Humedales de Costa Rica e Información Complementaria. SINAC, MINAE -UICN. Escala 1: 200000.

Breedy, O. \& H.M. Guzman. 2003. Octocorals from Costa Rica: The genus Pacifigorgia (Coelenterata: Octocorallia: Gorgoniidae). Zootaxa 281: 1-60.

Breedy, O. \& H.M. Guzman. 2005. A new species of Leptogorgia (Coelenterata: Ocotocorallia: Gorgoniidae) from the shallow waters of the eastern Pacific. Zootaxa 899: 1-11.

Breedy, O. \& H.M. Guzman. 2007. A revision of the genus Leptogorgia Milne Edwards \& Haime, 1857 (Coelenterata: Octocorallia: Gorgoniidae) in the eastern Pacific. Zootaxa 1407: 1-90.

Breedy, O. \& H.M. Guzman. 2011. A revision of the genus Heterogorgia Verrill, 1868 (Coelenterata: Octocorallia: Plexauridae. Zootaxa 2995: 27-44.

Brusca, R.C. \& E.W. Iverson. 1985. A guide to the marine isopod Crustacea of Pacific Costa Rica. Rev. Biol. Trop. 33 (Suppl. 1): 1-77.

Bussing, W.A. \& M. López. 2009. Marine fish. Texto: Pp. 453-458, Lista de especies, CD: Pp. 412-473. In: I.S. Wehrtmann \& J. Cortés (Eds.). Marine Biodiversity of Costa Rica, Central America. Springer, Berlín.

Cabrera-Peña, J. \& Y. Solano-López. 1996. Tamaños y frecuencia de Pontonia margarita (Crustacea: Palaemonidae) asociada a Pinctada mazatlanica (Bivalvia: Pteriidae), Costa Rica. Rev. Biol. Trop. 44: 915-917.

Cabrera-Peña, J., F. Vives-Jiménez \& Y. Solano-López. 1994. Tamaños y proporción sexual de Ucides occidentalis (Crustacea: Gecarcinidae) en el manglar un manglar de Costa Rica. Uniciencia 11: 97-99.

Castellanos, I.A., A. Morales-Ramírez \& E. Suárez-Morales. 2009. Appendicularians (Urochordata). Texto: Pp. 445-452, Lista de especies: Disco Compacto Pp. 411. In: I.S. Wehrtmann and J. Cortés (Eds.). Marine Biodiversity of Costa Rica, Central America. Springer, Berlín.

Castro, P. 1996. Eastern Pacific species of Trapezia (Crustacea, Brachyura: Trapeziidae), sibling species symbiotic with reef corals. Bull. Mar. Sci. 58: 531-554.

Cernohorsky, W.O. 1976. The Mitridae of the world, Part I: The Subfamily Mitrinae. Indo-Pac. Mollusca 3(17): 273-528.

Clark, H.L. 1940. Eastern Pacific expeditions of the New York Zoological Society. XXI. Notes on Echinoderms from the West Coast of Central America. Zoologica 25: 331-352.

Clark, H.L. 1948. A report on the Echini of the warmer eastern Pacific based on the collections of the Velero III. Allan Hancock Pac Exped. 8: 225-352.

Cortés, J. 2001. Requiem for an eastern Pacific seagrass bed, Bahía Culebra, Costa Rica. Rev. Biol. Trop. 49 (Suppl. 2): 273-278.

Cortés, J. \& H.M. Guzmán. 1998. Organismos de los arrecifes coralinos de Costa Rica: Descripción, distribución geográfica e historia natural de los corales zooxantelados (Anthozoa: Scleractinia) del Pacífico. Rev. Biol. Trop. 46: 55-91.

Cortés, J. \& C.E. Jiménez. 2003. Corals and coral reefs of the Pacific of Costa Rica: history, research and status: 361-385. In: J. Cortés (Ed.). Latin American Coral Reefs. Elsevier, Amsterdam.

Cortés, J. \& M.M. Murillo. 1985. Comunidades coralinas y arrecifes del Pacífico de Costa Rica. Rev. Biol. Trop. 33: 197-202.

Cortés, J., C.E. Jiménez, A.C. Fonseca \& J.J. Alvarado. 2010. Status and conservation of coral reefs in Costa Rica. Rev. Biol. Trop. 58 (Suppl. 1): 33-50.

Cortés, J., R. Vargas-Castillo \& J. Nivia. 2012. Marine biodiversity of Bahía Culebra, Guanacaste, Costa Rica: published records. Rev. Biol. Trop. 60 (Suppl. 2): 39-71.

Crane, J. 1941a. Eastern Pacific Expeditions of the New York Zoological Society. XXVI. Crabs of the Genus Uca from the west coast of Central America. Zoologica 26: 145-208.

Crane, J. 1941b. Eastern Pacific Expeditions of the New York Zoological Society. XXIX. On the growth and ecology of Brachyuran crabs of the Genus Ocypode. Zoologica 26: 297-310.

Crane, J. 1947. Eastern Pacific Expeditions of the New York Zoological Society. XXXVIII. Intertidal brachygnathous crabs from the west coast of tropical America with special reference to ecology. Zoologica 32: 69-95.

Crane, J. 1975. Fiddler Crabs of the World. Ocypodidae: Genus Uca. Princeton Univ. Press, Princeton, New Jersey. 738 p.

Crouch, R.W. \& C.W. Poag. 1987. Benthic Foraminifera of the Panamanian Province: distribution and origins. J. Foram. Res. 17: 153-176. 
Cushman, J.A. \& I. McCulloch. 1939. A report on some arenaceous Foraminifera. Allan Hancock Pac. Exped. 6: 1-113.

Cushman, J.A. \& I. McCulloch. 1940. Some Nonionidae in the Collections of the Allan Hancock Foundation. Allan Hancock Pac. Exped. 6: 145-178.

Cushman, J.A. \& I. McCulloch. 1942. Some Virgulininae in the Collections of the Allan Hancock Foundation. Allan Hancock Pac. Exped. 6: 179-230.

Cushman, J.A. \& I. McCulloch. 1948. The species of Bulimina and related genera in the collections of the Allan Hancock Foundation. Allan Hancock Pac. Exped. 6: 231-294.

Dawson, E.Y. 1957. Marine algae from the Pacific Costa Rican gulfs. Los Angeles County Mus. Contr. Sci. 15: $1-28$.

Dawson, E.Y. 1960. New records of marine algae from Pacific Mexico and Central America. Pac. Nat. 1(20): $31-52$.

Dawson, E.Y. 1961. A guide to the literature and distributions of Pacific benthic algae from Alaska to the Galapagos Islands. Pac. Sci. 15: 370-461.

Dawson, E.Y. \& P.T. Beaudette. 1959. Field notes from the 1959 eastern Pacific cruise of the Stella Polaris. Pac. Nat. 1(13): 1-24.

Dean, H.K. 2004. Marine biodiversity of Costa Rica: Class Polychaeta (Annelida). Rev. Biol. Trop. 52 (Suppl. 2): 131-181.

Deichmann, E. 1958. The Holothurioidea collected by the Velero III and IV during the years 1932 to 1954. Part II. Aspidochirota. Allan Hancock Pac. Exped. 11: 253-349.

Dominici-Arosemena, A. 1999. Estructura poblacional de los peces de arrecifes del Golfo de Papagayo, Guanacaste, Costa Rica, con énfasis en las especies de mayor importancia comercial como ornamentales. Tesis de Maestría, Univ. Costa Rica. San Pedro, Costa Rica. 208 p.

Dominici-Arosemena, A., E. Brugnoli-Olivera, J. CortésNúñez, H. Molina-Ureña \& M. Quesada-Alpízar. 2005. Community structure of eastern Pacific reef fishes (Gulf of Papagayo, Costa Rica). Tecnociencia 7(2): 19-41.

Drouet, F. 1936. Myxophyceae of the G. Allan Hancock Expedition of 1934, collected by Wm. R. Taylor. Allan Hancock Pac. Exp. 3: 15-32.
Fernández, C. \& J.J. Alvarado. 2008. Chlorophyta de la costa Pacífica de Costa Rica. Rev. Biol. Trop. 56 (Suppl. 4): 149-162.

Fernández, C. \& J. Cortés. 2005. Reef Site: Caulerpa sertularioides, a green alga spreading aggressively over coral reef communities in Culebra Bay, North Pacific of Costa Rica. Coral Reefs 24: 10 .

Fernández-García, C. 2007. Propagación del alga Caulerpa sertularioides (Chlorophyta) en Bahía Culebra, Golfo de Papagayo, Pacífico norte de Costa Rica. Tesis de Maestría en Biología, Universidad de Costa Rica. San Pedro, Costa Rica. 92 p.

Fradin, E. 1892. Estudios del Golfo de Nicoya, de la Bahía de Cocos y del Golfo de Culebra. Tipografía Nacional, San José: 97-106. (Re-impreso en Carlos Meléndez Ch. 1974. Viajes por Guanacaste. NOS VEN 4: 273-290).

Fraser, C.M. 1938. Hydroids of the 1934 Hancock Pacific Expeditions. Allan Hancock Pac. Exped. 4: 1-105.

Fraser, C.M. 1943a. General account of the scientific work of the Velero III in the eastern Pacific, 1931-41, Part I, Historical introduction, Velero III, personnel. Allan Hancock Pac. Exped. 1: 1-48.

Fraser, C.M. 1943b. General account of the scientific work of the Velero III in the eastern Pacific, 1931-1941, Part II: Geographic and biological associations. Allan Hancock Pac. Exped. 1: 49-258.

Fraser, C.M. 1943c. General account of the scientific work of the Velero III in the eastern Pacific, 1931-1941, Part III: a ten-year list of the Velero III collecting stations (Charts 1-115). With an appendix of collecting stations of the Allan Hancock Foundation for the year 1942. Allan Hancock Pac. Exped. 1: 259-431.

Fraser, C.M. 1948. Hydroids of the Allan Hancock Pacific Expeditions since March, 1938. Allan Hancock Pac. Exped. 4: 179-335.

Fritzsche, R.A. 1980. Revision of the Eastern Pacific Syngnathidae (Piscesisyngnathiformes), including both Recent and fossil forms. Proc. California Acad. Sci. 42: 181-227.

García, V., J. Acuña-González, J.A. Vargas-Zamora \& J. García-Céspedes. 2006. Calidad bacteriológica y desechos sólidos en cinco ambientes costeros de Costa Rica. Rev. Biol. Trop. 54 (Supl. 1): 35-48.

García-Céspedes, J., J.A. Acuña-González \& J.A. VargasZamora. 2004. Metales traza en sedimentos de cuatro ambientes costeros de Costa Rica. Rev. Biol. Trop. 52 (Supl. 2): 51-60. 
Garth, J.S. 1940. Some new species of Brachyuran crabs from Mexico and the Central and South American mainland. Allan Hancock Pac. Exped. 5: 53-127.

Garth, J.S. 1958. Brachyura of the Pacific coast of America. Oxyrhyncha. Tables and Plates. Allan Hancock Pac. Exped. 21: 501-854.

Garth, J.S. 1959. Eastern Pacific Expeditions of the New York Zoological Society. XLIV. Non-intertidial brachygnathous crabs from the west coast of Tropical America. Part 1: Brachygnatha, Oxyrhyncha. Zoologica 44: 105-126.

Garth, J.S. 1961. Eastern Pacific Expeditions of the New York Zoological Society. XLV. Non-intertidial brachygnathous crabs from the west coast of Tropical America. Part 2: Brachygnatha Brachyrhyncha. Zoologica 46: 133-160.

Garth, J.S. 1966. Eastern Pacific Expeditions of the New York Zoological Society. XLVI. Oxystomatous and allied crabs from the west coast of Tropical America. Zoologica 51: 1-16.

Gateño, D., A. León, Y. Barki, J. Cortés \& B. Rinkevich. 2003. Skeletal tumor formations in the massive coral Pavona clavus. Mar. Ecol. Prog. Ser. 258: 97-108.

Glynn, P.W., E.M. Druffel \& R.B. Dunbar. 1983. A dead Central American coral reef tract: possible link with the Little Ice Age. J. Mar. Res. 41: 605-637.

Glynn, P.W., N.J. Gassman, C.M. Eakin, J. Cortés, D.B. Smith \& H.M. Guzman. 1991. Reef coral reproduction in the eastern Pacific: Costa Rica, Panama, and Galápagos Islands (Ecuador), Part I-Pocilloporidae. Mar. Biol. 109: 355-368.

Glynn, P.W., S.B. Colley, C.M. Eakin, D.B. Smith, J. Cortés, N.J. Gassman, H.M. Guzman, J.B. del Rosario \& J. Feingold. 1994. Reef coral reproduction in the eastern Pacific: Costa Rica, Panama, and Galápagos Islands (Ecuador) - II. Poritidae. Mar. Biol. 118: 191-208.

Glynn, P.W., S.B. Colley, N.J Gassman, K. Black, J. Cortés \& J.L. Maté. 1996. Reef coral reproduction in the eastern Pacific: Costa Rica, Panama, and Galápagos Islands (Ecuador) - III. Agariciidae (Pavona gigantea and Gardineroseris planulata). Mar. Biol. 125: 579-601.

Glynn, P.W., S.B. Colley, J.H Ting, J.L. Maté \& H.M. Guzman. 2000. Reef coral reproduction in the eastern Pacific: Costa Rica, Panama, and Galápagos Islands (Ecuador) - IV. Agariciidae, recruitment and recovery of Pavona varians and Pavona sp.a. Mar. Biol. 136: 785-805.
Glynn, P.W., S.B. Colley, J.L. Maté, J. Cortés, H.M. Guzmán, R.L. Bailey, J.S Feingold \& I.C. Enochs. 2008. Reproductive ecology of the azooxanthellate coral Tubastrea coccinea_in the Equatorial Eastern Pacific: Part V. Dendrophyllidae. Mar. Biol. 153: 529-544.

Glynn, P.W., S.B. Colley, H.M. Guzman, I.C. Enochs, J. Cortés, J.L. Maté \& J.S. Feingold. 2011. Reef coral reproduction in the eastern Pacific: Costa Rica, Panamá and the Galápagos Islands (Ecuador). VI. Agariciidae, Pavona clavus. Mar. Biol. 158: 1601-1617.

Gore, R. 1982. Porcellanid crabs from the coasts of Mexico and Central America (Crustacea: Decapoda: Anomura). Smithsonian Contr. Zool. 363: 1-34.

Gould, C.G. 2004. The Remarkable Life of William Beebe: Explorer and Naturalist. Island Press, Washington, Covelo, London.

Gravel, P., K. Johanning, J. McLachlan, J.A. Vargas \& E. Oberdörster. 2006. Imposex in the intertidal snail Thais brevidentata (Gastropoda: Muricidae) from the Pacific coast of Costa Rica. Rev. Biol. Trop. 54 (Suppl. 1): 21-26.

Haig, J. 1960. The Porcellanidae (Crustacea: Anomura) of the eastern Pacific. Allan Hancock Pac. Exped. 24: $1-440$.

Haig, J. 1968. Eastern Pacific expeditions of the New York Zoological Society. Porcellanid crabs (Crustacea: Anomura) from the west coast of Tropical America. Zoologica 53: 57-74.

Hanna, G.D. \& A.M. Strong. 1949. West American mollusks of the genus Conus. Proc. Calif. Acad. Sci. 4th Ser., 26: 247-322.

Hartman, O. 1940. Polychaetous Annelids. Part II. Chrysopetalidae to Goniadidae. Allan Hancock Pac. Exped. 7: 173-287.

Hartman, O. 1944a. Polychaetous annelids. Pt. V. Eunicea. Allan Hancock Pac. Exped. 10: 1-238.

Hartman, O. 1944b. Polychaetous annelids. Pt. VI. Paraonidae, Magelonidae, Longosomidae, Ctenodrilidae and Sabellariidae. Allan Hancock Pac. Exped. 10: 311-389.

Hendrickx, M.E. \& M.K. Wicksten. 1989. Los Pandalidae (Crustacea: Caridea) del Pacífico mexicano, con una clave para su identificación. Caldasia 16: 71-86.

Henry, D.P. \& P.A. McLaughlin. 1967. A revision of the subgenus Solidabalanus Hoek (Cirripedia, Thoracica) 
including a description of a new species with complemental males. Crustaceana 12: 43-58.

Henry, D.P. \& P.A. McLaughlin. 1975. The barnacles of the Balanus amphitrite complex (Cirripedia, Thoracica). Zool. Verh. 141: 1-254.

Hertlein, L.G. \& A.M. Strong. 1943. Eastern Pacific Expeditions of the New York Zoological Society. XXXII. Mollusks from the West Coast of Mexico and Central America. Part II. Zoologica 28: 149-168.

Hertlein, L.G. \& A.M. Strong. 1946a. Eastern Pacific Expeditions of the New York Zoological Society. XXXIV. Mollusks from the West Coast of Mexico and Central America. Part III. Zoologica 31: 53-76.

Hertlein, L.G. \& A.M. Strong. 1946b. Eastern Pacific Expeditions of the New York Zoological Society. XXXV. Mollusks from the West Coast of Mexico and Central America. Part IV. Zoologica, 31: 93-120.

Hertlein, L.G. \& A.M. Strong. 1947. Eastern Pacific Expeditions of the New York Zoological Society. XXXVI. Mollusks from the West Coast of Mexico and Central America. Part V. Zoologica 31: 129-150.

Hertlein, L.G. \& A.M. Strong. 1948. Eastern Pacific Expeditions of the New York Zoological Society. XXXIX. Mollusks from the West Coast of Mexico and Central America. Part VI. Zoologica 33: 163-198.

Hertlein, L.G. \& A.M. Strong. 1949a. Eastern Pacific Expeditions of the New York Zoological Society. XL. Mollusks from the West Coast of Mexico and Central America. Part VII. Zoologica 34: 63-97.

Hertlein, L.G. \& A.M. Strong. 1949b. Eastern Pacific Expeditions of the New York Zoological Society. XLI. Mollusks from the West Coast of Mexico and Central America. Part VIII. Zoologica 34: 239-258.

Hertlein, L.G. \& A.M. Strong. 1950. Eastern Pacific Expeditions of the New York Zoological Society. XLII. Mollusks from the West Coast of Mexico and Central America. Part IX. Zoologica 35: 217-252.

Hertlein, L.G. \& A.M. Strong. 1951. Eastern Pacific Expeditions of the New York Zoological Society. XLIII. Mollusks from the West Coast of Mexico and Central America. Part X. Zoologica 36: 66-120.

Hertz, C.M., B.W. Myers \& J. Gemmell. 1992. Two new vitrinellid species from the Gulf of California, Mexico (Gastropoda: Vitrinellidae). Veliger 35: 70-73.

Hoberg, E.P., D.R. Brooks, H. Molina-Ureña \& E. Erbe. 1998. Echinocephalus janzeni n.sp. (Nematoda: Gnathostomatidae) in Himantura pacifica
(Chondrichthyes: Myliobatiformes) from the Pacific coast of Costa Rica and Mexico, with historial biogeographic analysis of the genus. J. Parasitol. 84: 571-581.

Ibarra-Gene, E. 2006. El valor de uso del paisaje submarino en el Golfo de Papagayo: comparación de la industria de buceo deportivo con la industria de extracción de peces de acuario. Licenciatura en Economía, Facultad de Ciencias Económicas, Universidad de Costa Rica. San Pedro, Costa Rica. 155 p.

Jiménez, C.E. 1997. Corals and coral reefs of Culebra Bay, Pacific coast of Costa Rica: Anarchy in the reef. Proc. 8th Int. Coral Reef Symp., Panamá 1: 329-334.

Jiménez, C.E. 1998. Arrecifes y comunidades coralinas de Bahía Culebra, Pacifico Norte de Costa Rica (Golfo de Papagayo). Tesis de Maestría en Biología, Universidad de Costa Rica. San Pedro, Costa Rica. 218 p.

Jiménez, C. 2001a. Seawater temperature measured at the surface and at two depths (7 and $12 \mathrm{~m}$ ) in one coral reef at Culebra Bay, Gulf of Papagayo, Costa Rica. Rev. Biol. Trop. 49 (Suppl. 2): 153-161.

Jiménez, C. 2001b. Arrecifes y ambientes coralinos de Bahía Culebra, Pacífico de Costa Rica: aspectos biológicos, económico-recreativos y de manejo. Rev. Biol. Trop. 49 (Supl. 2): 215-231.

Jiménez, C.E. \& J. Cortés. 2003. Growth of seven species of scleractinian corals in an upwelling environment of the eastern Pacific (Golfo de Papagayo, Costa Rica). Bull. Mar. Sci. 72: 187-198.

Jiménez, C., J. Cortés, A. León \& E. Ruiz. 2001. Coral bleaching and mortality associated with the1997-98 El Niño in an upwelling environment in the eastern Pacific (Gulf of Papagayo, Costa Rica). Bull. Mar. Sci. 69: 151-169.

Jiménez, C., G. Bassey, Á. Segura \& J. Cortés. 2010. Characterization of the coral communities and reefs of two previously undescribed locations in the upwelling region of Gulf of Papagayo (Costa Rica). Rev. Cienc. Mar. Cost. 2: 95-108.

Jung, P. 1989. Revision of the Strombina-Group (Gastropoda: Columbellidae), Fossil and Living: Distribution, Biostratigraphy and Systematics. Schweiz. Paläont. Abhandl. 111: 1-298.

Kelmo, F. \& R. Vargas. 2002. Anthoathecatae and Leptothecatae hydroids from Costa Rica (Cnidaria: Hydrozoa). Rev. Biol. Trop. 50: 599-627. 
Kim, W. \& L.G. Abele. 1988. The snapping shrimp genus Alpheus from the eastern Pacific (Decapoda: Caridea: Alpheidae). Smithson. Contr. Zool. 454: 1-119.

Lalicker, C.G. \& I. McCulloch. 1940. Some Textulariidae of the Pacific Ocean. Allan Hancock Pac. Exped. 6: 115-143.

Lizano, O.G., L.G. Loría, E.J. Alfaro \& M. Badilla. 2008 Distribución espacial de radionucleídos en sedimentos marinos de Bahía Culebra y el Golfo de Nicoya, Pacífico, Costa Rica. Rev. Biol. Trop. 56 (Supl. 4): 83-90.

Madrigal-Castro, E., J. Cabrera-Peña, J. Monge-Esquivel \& F. Pérez-Acuña. 1984. Comparación entre dos poblaciones de Acanthina brevidentata (Gastropoda: Mollusca) en dos zonas rocosas de Playa Panamá, Guanacaste, Costa Rica. Rev. Biol. Trop. 32: 11-15.

Manning, R.B. 1971. Eastern Pacific Expeditions of the New York Zoological Society. Stomatopod Crustacea. Zoologica 56: 95-113.

Manter, H.W. 1940. Digenetic trematodes of fishes from the Galapagos Islands and the neighboring Pacific. Allan Hancock Pac. Exped. 2: 329-497.

Marques, F., D.R. Brooks \& H. Molina-Ureña. 1996. Two new species of Tetraphyllidean Cestodes in Himantura pacifica (Chondrichthyes: Myliobatiformes: Dasyatididae) from the northwest Coast of Costa Rica. J. Parasitol. 82: 302-306.

May-Collado, L.J. 2001. Ecología y comportamiento del delfín manchado costero, Stenella attenuata graffmani (CETACEA: DELPHINIDAE) del Pacifico norte de Costa Rica. Tesis de Maestría, Univ. Costa Rica. San Pedro, Costa Rica. 78 p.

May-Collado, L. 2009. Marine mammals. Texto: Pp. 479495, Lista de especies, CD: Pp. 485-490. In: I.S. Wehrtmann \& J. Cortés (Eds.). Marine Biodiversity of Costa Rica, Central America. Springer, Berlín.

May-Collado, L. \& A. Morales-Ramírez. 2005. Presencia y patrones de comportamiento del delfín manchado costero, Stenella attenuata (Cetacea: Delhinidae) en el Golfo de Papagayo, Costa Rica. Rev. Biol. Trop. 53: $265-276$.

May-Collado, L., T. Gerrodette, J. Calambokidis, K. Rasmussen \& I. Sereg. 2005. Patterns of cetacean sighting distribution in the Pacific Exclusive Economic Zone of Costa Rica based on data collected from 1979-2001. Rev. Biol. Trop 53: 249-263.

McLaughlin, P.A. 1981. Revision of Pylopagurus and Tomopagurus (Crustacea: Decapoda: Paguridae), with the descriptions of new genera and species: Part II. Rhodochirus McLaughlin and Phimochirus McLaughlin. Bull. Mar. Sci. 31: 329-365.

McLean, J.H. \& R. Poorman. 1971. New species of tropical eastern Pacific Turridae. Veliger 14: 89-113.

Meserve, F.G. 1938. Some monogenetic trematodes from the Galapagos Islands and the neighboring Pacific. Allan Hancock Pac. Exped. 2: 31-89.

Morales-Ramírez, A., R. Víquez, K. Rodríguez \& M. Vargas. 2001. Marea roja producida por Lingulodinium polyedrum (Peridiniales, Dinophyceae) en Bahía Culebra, Golfo de Papagayo, Costa Rica. Rev. Biol. Trop. 49 (Supl. 2): 19-23.

Muller-Parker, G. \& J. Cortés. 2001. Spatial distribution of light and nutrients in some coral reefs of Costa Rica during January 1997. Rev. Biol. Trop. 49 (Suppl. 2): 251-263.

Osburn, R.C. 1950. Bryozoa of the Pacific Coast of America. Part I, Cheilostomata-Anasca. Allan Hancock Pac. Exped. 14: 1-269.

Osburn, R.C. 1952. Bryozoa of the Pacific Coast of America. Part II, Cheilostomata-Ascophora. Allan Hancock Pac. Exped. 14: 271-611.

Osburn, R.C. \& J.D. Soule. 1953. Bryozoa of the Pacific Coast of America. Part III, Order Ectoprocta. Allan Hancock Pac. Exped. 14: 726-758.

Pérez-Ponce de León, G., V. León-Regagnon \& S. Monks. 1998. Theletrum lamothei sp. nov. (Digenea), parasite of Echidna nocturna from Cuajiniquil, Guanacaste, and other digenes of marine fishes from Costa Rica. Rev. Biol. Trop. 46: 345-354.

Pfeiler, E. 2008. Resurrection of the name Albula pacifica (Beebe, 1942) for the shafted bonefish (Albuniformes: Albulidae) from the eastern Pacific. Rev. Biol. Trop. 56: 839-844.

Rathbun, M.J. 1937. The Oxystomatous and allied crabs of America. U.S. Natl. Mus. Bull. 166: 1-272.

Reid, D.G. 2002. The genus Nodilittorina von Martens, 1897 (Gastropoda: Littorinidae) in the eastern Pacific Ocean, with a discussion of Biogeographic Provinces of the rocky-shore fauna. Veliger 45: 85-170.

Ritson-Williams, R., S.N. Arnold, N.D. Fogarty, R.S. Steneck, M.J.A. Vermeij \& V.J. Paul. 2009. New perspectives on ecological mechanisms affecting coral recruitment on reefs. Smithson. Contr. Mar. Sci. 38: 437-457. 
Rodríguez-Ortíz, B., L. García-Prieto \& G. Pérez-Ponce de León. 2004. Checklist of the helminth parasites of vertebrates in Costa Rica. Rev. Biol. Trop. 52: 313-354.

Rodríguez-Sáenz, K.E. 2005. Distribución espacial y temporal de la biomasa, composición y abundancia del zooplancton, con énfasis en hidromedusas de Bahía Culebra, durante La Niña 1999 y el 2000. Tesis de Maestría en Biología, Universidad de Costa Rica. San Pedro, Costa Rica. 156 p.

Rodríguez-Sáenz, K. \& R. Gasca. 2009. Siphonophores. Texto: Pp. 151-156, Lista de especies, CD: Pp. 101-104. In: I.S. Wehrtmann \& J. Cortés (Eds.). Marine Biodiversity of Costa Rica, Central America. Springer, Berlín.

Rodríguez-Sáenz, K. \& J. Rodríguez-Fonseca. 2004. Avistamientos del delfín manchado, Stenella attenuata (Cetacea: Delphinidae) en Bahía Culebra, Costa Rica, 1999-2000. Rev. Biol. Trop. 52 (Supl. 2): 189-193.

Rodríguez-Sáenz, K. \& L. Segura-Puertas. 2009. Hydrozoa, Scyphozoa, and Cubozoa (Medusozoa). Texto: Pp. 143-149, Lista de especies, CD: Pp. 94-100. In: I.S. Wehrtmann \& J. Cortés (Eds.). Marine Biodiversity of Costa Rica, Central America. Springer, Berlín.

Rost, H. 1955. A report on the Family Arcidae (Pelecypoda). Allan Hancock Pac. Exped. 20: 177-249.

Samper-Villareal, J., J. Cortés \& C. Benavides. 2012. Description of the Panamá and Iguanita mangrove stands within Bahia Culebra, north Pacific coast of Costa Rica. Rev. Biol. Trop. 60 (Supl. 2): 109-120.

Savage, J.M. 2002. The Amphibians and Reptiles of Costa Rica: A Herpetofauna between Two Continents, between Two Seas. Univ. Chicago Press, Chicago, London. 934 p.

Schmitt, W.L. 1940. The Stomatopods of the West Coast of America, based on collections made by the Allan Hancock Expeditions, 1933-38. Allan Hancock Pac. Exped. 5: 129-225.

Skoglund, C. 2001. Panamic Province Molluscan Literature. Additions and changes from 1971 through 2000. I. Bivalvia. Festivus 32 (Supplement): 119 p.

Skoglund, C. 2002. Panamic Province Molluscan Literature. Additions and changes from 1971 through 2001. III Gastropoda. Festivus 33 (Supplement): 286 p.

Soot-Ryen, T. 1955. A report on the Family Mytilidae (Pelecypoda). Allan Hancock Pac. Exped. 20: 1-175.
Spongberg, A.L. 2004. PCB Contamination in surface sediments in the coastal waters of Costa Rica. Rev. Biol. Trop. 52 (Suppl. 2): 1-10.

Spongberg, A.L. 2006. PCB concentrations in intertidal sipunculan (Phylum Sipuncula) marine worms from the Pacific coast of Costa Rica. Rev. Biol. Trop. 54 (Suppl. 1): 27-33.

Suárez-Morales, E. \& A. Morales-Ramírez. 2003. A new species of Cymbasoma (Crustacea: Copepoda: Monstrilloida) from the Pacific coast of Costa Rica, Central America. Proc. Biol. Soc. Wash. 116: 206-214.

Sunagawa, S. 2005. Seasonal Variation in Symbiont Densities and Skeletal Phosphorus Concentrations in the Eastern Pacific (Costa Rica) Coral Pavona clavus. Tesis M.Sc., ISATEC, Univ. Bremen, Alemania.

Sunagawa, S., J. Cortés, C. Jiménez \& R. Lara. 2008. Variación en la densidad de células y en las concentraciones de pigmentos de los dinoflagelados simbióticos del coral Pavona clavus en el Pacífico oriental (Costa Rica). Cienc. Mar. 34: 113-123.

Svendsen, H., R. Rosland, S. Myking, J.A. Vargas, O.G. Lizano \& E.J. Alfaro. 2006. A physical-oceanographic study of Golfo Dulce, Costa Rica. Rev. Biol. Trop. 54 (Suppl. 1): 147-170.

Tarrant, A.M., J. Cortés, M. Atkinson, S. Atkinson, K. Johanning, T.-c. Chiang, J.A. Vargas \& J.A. McLachlan. 2008. Three orphan nuclear receptors in the scleractinian coral Pocillopora damicornis from the Pacific coast of Costa Rica. Rev. Biol. Trop. 56 (Suppl. 4): 39-48.

Taylor, E.H. 1953. Early records of the seasnake Pelamis platurus in Latin America. Copeia 1953: 124.

Taylor, W.R. 1945. Pacific marine algae of the Allan Hancock Pacific Expeditions to the Galapagos Islands. Allan Hancock Pacific Exped. 12: 1-528.

Tsuda, R.T. 1968. Additional records of the marine algae from Costa Rica. Carib. J. Sci. 8: 103-104.

Tu, A.T. 1976. Investigation of the sea snake, Pelamis platurus (Reptilia, Serpentes, Hydrophiidae), on the Pacific coast of Costa Rica, Central America. J. Herpetol. 10: 13-18.

Türkay, M. 1970. Die Gecarcinidae Amerikas. Mit einem Anhang über Ucides Rathbun. Sencken. Biol. 51: 333-354.

Van Cleave, H.J. 1940. The Acanthocephala collected by the Allan Hancock Pacific Expedition, 1934. Allan Hancock Pac. Exped. 2: 501-527. 
Valdés, Á. \& Y.E. Camacho-García. 2004. “Cephalaspidean" Heterobranchs (Gastropoda) from the Pacific Coast of Costa Rica. Proc. California Acad. Sci. 55: 459-497.

Vargas-Castillo, R. 2012. Nuevas adiciones a la fauna de crustáceos decápodos de Bahía Culebra, Guanacaste, Costa Rica. Rev. Biol. Trop. 60 (Supl. 2): 73-76.

Vargas, R. \& J. Cortés. 2006. Biodiversidad marina de Costa Rica: Crustacea: Infraorden Anomura. Rev. Biol. Trop. 54: 461-488.

Vargas-Montero, M. 2004. Floraciones algales en Costa Rica y su relación con algunos factores meteorológicos y consideraciones sobre sus efectos socioeconómicos. Tesis de Maestría, GIACT, Univ. Costa Rica. San Pedro, Costa Rica. 90 p.

Wehrtmann, I.S. \& R. Vargas. 2003. New records and range extensions of shrimps (Decapoda: Penaeoidea, Caridea) from the Pacific and Caribbean coasts of Costa Rica, Central America. Rev. Biol. Trop. 51: 268-274.

Wicksten, M.K. 1983. Shallow water caridean shrimps of the Gulf of California, México. Allan Hancock Monogr. Mar. Biol. 13: 1-59.
Wicksten, M.K. \& M.E. Hendrickx. 1992. An updated checklist of benthic marine and brackish water shrimps (Decapoda: Penaeoidea, Stenopodidea, Caridea) from the Eastern Tropical Pacific: 49-76. In: M.E. Hendrickx (ed.), Contribuciones al estudio de los Crustáceos del Pacífico Este, 2. Instituto de Ciencias del Mar y Limnología, UNAM.

Wilson, C.B. 1937. Parasitic copepods taken during the third Hancock expedition to the Galapagos Islands. Allan Hancock Pac. Exped. 2: 23-31.

Wolff, M., H.J. Hartmann \& V. Koch. 1996. A pilot trophic model for Golfo Dulce, a fjord-like tropical embayment, Costa Rica. Rev. Biol. Trop. 44 (Suppl. 3): 215-231.

Wolff, M., J. Chavarría, V. Koch \& J.A. Vargas. 1998. A trophic flow model of the Golfo de Nicoya, Costa Rica. Rev. Biol. Trop. 46 (Suppl. 6): 63-79.

Zamora-Trejos, P. \& J. Cortés. 2009. Los manglares de Costa Rica: Pacífico norte. Rev. Biol. Trop. 57: 473-488.

Ziesenhenne, F.C. 1940. New ophiurans of the Allan Hancock Pacific Expeditions. Allan Hancock Pac. Exped. 8: 9-59. 
